\title{
Stability of plane-wave solutions of a dissipative generalization of the nonlinear Schrödinger equation
}

\author{
John D. Carter \\ Mathematics Department \\ Seattle University \\ 901 12th Avenue \\ Seattle, WA 98122 \\ Cynthia C. Contreras \\ Display Technologies \\ Corning, Incorporated \\ Corning, NY 14831
}

\begin{abstract}
The modulational instability of perturbed plane-wave solutions of the cubic nonlinear Schrödinger (NLS) equation is examined in the presence of three forms of dissipation. We present three families of decreasing-in-magnitude plane-wave solutions to this dissipative NLS equation. We establish that all such solutions that have no spatial dependence are linearly stable, though some perturbations may grow a finite amount. Further, we establish that all such solutions that have spatial dependence are linearly unstable if a certain form of dissipation is present.
\end{abstract}

\section{Introduction}

The (2+1)-dimensional cubic nonlinear Schrödinger (NLS) equation is given by

$$
i \psi_{t}+\alpha \psi_{x x}+\beta \psi_{y y}+\gamma|\psi|^{2} \psi=0
$$

where $\alpha, \beta$, and $\gamma$ are real constants, $\psi=\psi(x, y, t)$ is a complex-valued function, and the subscripts represent partial derivatives. Among many other

Email addresses: carterj1@seattleu.edu (John D. Carter), contreracc@corning.com (Cynthia C. Contreras). 
physical situations, the NLS equation arises as an approximate model of the evolution of a nearly monochromatic wave of small amplitude in pulse propagation along optical fibers $(\alpha \beta>0)$ [16], in gravity waves on deep water $(\alpha \beta<0)[3,11]$, and in Langmuir waves in a plasma $(\alpha \beta>0)$ [19]. As a description of a Bose-Einstein condensate, the NLS is known as the GrossPitaevskii equation $(\alpha \beta>0)[20,14]$.

The NLS equation does not contain any terms that represent dissipation. There are many generalizations of the NLS equation that contain dissipative terms including those presented in [17, 4, 21, 22, 24]. We consider the following equation which we refer to as the dissipative nonlinear Schrödinger (DissNLS) equation,

$$
i \psi_{t}+(\alpha-i a) \psi_{x x}+(\beta-i b) \psi_{y y}+(\gamma+i c)|\psi|^{2} \psi+i d \psi=0
$$

Here $\psi=\psi(x, y, t)$ is a complex-valued function, $\alpha, \beta, \gamma, a, b, c$, and $d$ are real constants with $a, b, c$, and $d$ all nonnegative. Davey [10] gives a general argument for dissipative terms of the forms given in equation (2) when the NLS equation is used as a model of weakly nonlinear surface waves. Lake et al. [15] generalize the NLS equation to include dissipation in a physical system by adding the $i d \psi$ term. Blennerhassett [4] derives an equation similar to equation (2) from the Navier-Stokes' equations for a free surface flow with viscous free surface boundary conditions. Segur et al. [22] add the $i d \psi$ term in order to account for the effects of weak dissipation. They show strong agreement between the mathematical theory and a series of physical experiments. Our work is motivated by Bridges \& Dias [5] in which equation (2) is examined from a different mathematical perspective.

Equation (2) can also be thought of as a generalization of the complex GinzburgLandau (CGL) equation $[13,2]$. Note that we require $d \geq 0$, while $d<0$ in the most common form of the CGL equation. All quantities in equations (1) and (2) are dimensionless. We consider both the NLS and DissNLS equations with periodic boundary conditions.

The NLS equation conserves (in $t$ ) the $L_{\text {per }}^{2}$ norm,

$$
\|\psi\|_{L_{\mathrm{per}}^{2}}^{2}=\int_{0}^{L_{y}} \int_{0}^{L_{x}}|\psi|^{2} d x d y
$$

where $L_{x}$ and $L_{y}$ are the periods of $\psi$ in the $x$ - and $y$-dimensions respectively. In general, the DissNLS equation does not conserve the $L_{\text {per }}^{2}$ norm. Specifically, the $L_{\text {per }}^{2}$ norm is nonincreasing in $t$ and

$$
\frac{d}{d t}\left(\|\psi\|_{L_{\mathrm{per}}^{2}}^{2}\right)=-2 \int_{0}^{L_{y}} \int_{0}^{L_{x}}\left(a\left|\psi_{x}\right|^{2}+b\left|\psi_{y}\right|^{2}+c|\psi|^{4}+d|\psi|^{2}\right) d x d y .
$$




\subsection{Solutions}

The NLS equation admits plane-wave solutions of the form

$$
\psi(x, y, t)=\psi_{0} \exp \left(i k x+i l y+i\left(-\alpha k^{2}-\beta l^{2}+\gamma \psi_{0}^{2}\right) t+i \xi\right),
$$

where $\psi_{0}, k, l$, and $\xi$ are real constants. The magnitude of any such solution is constant.

The DissNLS equation admits four families of plane-wave solutions of the form

$$
\psi(x, y, t)=\psi_{0} \exp \left(i k x+i l y+\omega_{r}(t)+i \omega_{i}(t)+i \xi\right),
$$

where $\psi_{0}, k, l$, and $\xi$ are real constants, and $\omega_{r}(t)$ and $\omega_{i}(t)$ are real-valued functions. Substituting equation (6) into equation (2), separating into real and imaginary parts, and simplifying gives

$$
\begin{gathered}
\omega_{r}^{\prime}+a k^{2}+b l^{2}+d+c \psi_{0}^{2} \mathrm{e}^{2 \omega_{r}}=0, \\
\omega_{i}^{\prime}+\alpha k^{2}+\beta l^{2}-\gamma \psi_{0}^{2} \mathrm{e}^{2 \omega_{r}}=0,
\end{gathered}
$$

where prime represents derivative with respect to $t$. This system admits four different solutions depending on the parameter values. In each of the following cases, the constants of integration were chosen so that these solutions limit to the plane-wave solutions of the NLS equation as $a, b, c, d$ limit to zero.

- Case 1: $\quad c=0$ and $a k^{2}+b l^{2}+d=0$

The solution of the system of equations given in equation (7) is

$$
\begin{gathered}
\omega_{r}(t)=0, \\
\omega_{i}(t)=-t\left(\alpha k^{2}+\beta l^{2}-\gamma \psi_{0}^{2}\right) .
\end{gathered}
$$

The magnitude of any of these solutions is constant in $t$. The NLS solutions given in equation (5) are a subset of these solutions.

- Case 2: $\quad c>0$ and $a k^{2}+b l^{2}+d=0$

The solution is

$$
\begin{gathered}
\omega_{r}(t)=-\frac{1}{2} \ln \left(1+2 c \psi_{0}^{2} t\right), \\
\omega_{i}(t)=-t\left(\alpha k^{2}+\beta l^{2}\right)-\frac{\gamma}{c} \omega_{r}(t) .
\end{gathered}
$$

The magnitude of any of these solutions decays to zero as $t^{-1 / 2}$.

- Case 3: $\quad c=0$ and $a k^{2}+b l^{2}+d>0$

The solution is

$$
\begin{gathered}
\omega_{r}(t)=-t\left(a k^{2}+b l^{2}+d\right), \\
\omega_{i}(t)=-t\left(\alpha k^{2}+\beta l^{2}\right)+\frac{\gamma \psi_{0}^{2}}{2\left(a k^{2}+b l^{2}+d\right)}\left(1-\mathrm{e}^{-2 t\left(a k^{2}+b l^{2}+d\right)}\right) .
\end{gathered}
$$

The magnitude of any of these solutions decays to zero exponentially. 
- Case 4: $\quad c>0$ and $a k^{2}+b l^{2}+d>0$

The solution is

$$
\begin{aligned}
& \omega_{r}(t)=\frac{1}{2} \ln \left(\frac{a k^{2}+b l^{2}+d}{\left(a k^{2}+b l^{2}+d+c \psi_{0}^{2}\right) \mathrm{e}^{2 t\left(a k^{2}+b l^{2}+d\right)}-c \psi_{0}^{2}}\right), \\
& \omega_{i}(t)=-t\left(\alpha k^{2}+\beta l^{2}+\frac{\gamma}{c}\left(a k+b l^{2}+d\right)\right)-\frac{\gamma}{c} \omega_{r}(t) .
\end{aligned}
$$

The magnitude of any of these solutions decays to zero exponentially.

If $a>0$, then the magnitude of DissNLS solutions of the form given in equation (6) with $k \neq 0$ decays to zero faster than the magnitude of solutions with $k=0$. Similarly, if $b>0$, then the magnitude of DissNLS solutions of the form given in equation (6) with $l \neq 0$ decays to zero faster than the magnitude of solutions with $l=0$. These differences between spatially-independent and spatially-dependent solutions are related to equation (4) and play an important role in the calculations below.

In Sections 2.1-2.3, we establish that if $c$ and/or $d$ is positive, then all spatiallyindependent plane-wave solutions of the DissNLS equation are linearly stable regardless of the signs of $\alpha, \beta$, and $\gamma$. We establish that if $a>0$ and $b>0$, then all spatially-dependent plane-wave solutions of the DissNLS equation are linearly unstable regardless of the signs of $\alpha, \beta$, and $\gamma$. Further, we establish that the most unstable mode is directly related to the spatially-independent plane-wave solution. We corroborate these results numerically in Section 3.

\section{Stability analysis}

The stability of plane-wave solutions of the CGL equation with $d<0$ has been well studied $[18,23,6,2]$. To our knowledge the stability of decreasingin-amplitude plane-wave solutions of the DissNLS equation of the forms given in cases 2 through 4 has not been examined in as much detail.

\subsection{Case 1: $\quad c=0$ and $a k^{2}+b l^{2}+d=0$}

In order to study the linear stability of solutions of the form given in equation (6) with $\omega_{r}(t)$ and $\omega_{i}(t)$ defined in equation (8), we consider perturbed solutions of the form

$$
\begin{gathered}
\psi_{p}(x, y, t)=\left(\psi_{0}+\epsilon u(x, y, t)+i \epsilon v(x, y, t)+\mathcal{O}\left(\epsilon^{2}\right)\right) * \\
\exp \left(i k x+i l y-i t\left(\alpha k^{2}+\beta l^{2}-\gamma \psi_{0}^{2}\right)+i \xi\right),
\end{gathered}
$$


where $\epsilon$ is a small real parameter and $u(x, y, t)$ and $v(x, y, t)$ are real-valued functions. This case is a (minor) generalization of the work by Zakharov [26] and Davey \& Stewartson [11] in which the stability of plane-wave solutions of the NLS equation is examined.

Substituting equation (12) into equation (2), linearizing, and separating into real and imaginary parts leads to a pair of coupled, constant-coefficient partial differential equations (PDEs). Without loss of generality, assume

$$
\begin{aligned}
& u(x, y, t)=\bar{U} \mathrm{e}^{i p x+i q y+\Omega t}+c . c . \\
& v(x, y, t)=\bar{V} \mathrm{e}^{i p x+i q y+\Omega t}+c . c .
\end{aligned}
$$

where $\bar{U}, \bar{V}$, and $\Omega$ are complex constants, $p$ and $q$ are real constants, and c.c. denotes complex conjugate. If $\Omega$ has a positive real part, then the perturbations $u$ and $v$ grow exponentially in $t$ and the unperturbed solution is said to be linearly unstable. After substituting equation (13) into the PDEs, the system simplifies to the following pair of algebraic equations

$$
\left(\begin{array}{cc}
2 \gamma \psi_{0}^{2}-\alpha p^{2}-\beta q^{2} & -\Omega-a p^{2}-b q^{2}-2 i(\alpha k p+\beta l q) \\
\Omega+a p^{2}+b q^{2}+2 i(\alpha k p+\beta l q) & -\alpha p^{2}-\beta q^{2}
\end{array}\right)\left(\begin{array}{l}
\bar{U} \\
\bar{V}
\end{array}\right)=\left(\begin{array}{l}
0 \\
0
\end{array}\right) .
$$

In order for this system to have a nonzero solution, $\Omega$ must be chosen to satisfy

$$
\Omega_{ \pm}=-a p^{2}-b q^{2}-2 i(\alpha k p+\beta l q) \pm \sqrt{\left(\alpha p^{2}+\beta q^{2}\right)\left(2 \gamma \psi_{0}^{2}-\alpha p^{2}-\beta q^{2}\right)}
$$

- $\Re\left(\Omega_{ \pm}\right)$does not depend on $k$ or $l$. $(\Re(\Omega)$ denotes the real part of $\Omega$.) This establishes that the spatial dependence of the unperturbed solution plays no role in the growth rate of this instability.

- If $\gamma=0$ or if $(\alpha \beta \geq 0$ and $(\alpha \gamma<0$ or $\beta \gamma<0))$ then the term under the square root in equation (15) is negative and thus $\Re\left(\Omega_{-}\right)=\Re\left(\Omega_{+}\right) \leq 0$. Therefore all corresponding solutions are linearly stable.

- In the NLS case, for all other choices of $\alpha, \beta$, and $\gamma$ there exists a region of $(p, q)$-space for which $\Omega_{+}$has a positive real part. Therefore all corresponding solutions are linearly unstable. The maximal growth rate (i.e. the maximal real part of $\left.\Omega_{+}\right)$is $|\gamma| \psi_{0}^{2}$.

- In the DissNLS case, for all other choices of $\alpha, \beta$, and $\gamma$, there exists a region of $(p, q)$-space for which the term under the square root is positive. This establishes $\Re\left(\Omega_{-}\right)<\Re\left(\Omega_{+}\right)$with $\Re\left(\Omega_{-}\right)<0$. There may or may not be instability depending on the parameter values. However, any instability that arises from the DissNLS equation in this case has a maximal growth rate less than or equal to $|\gamma| \psi_{0}^{2}$. 


\subsection{Case 2: $\quad c>0$ and $a k^{2}+b l^{2}+d=0$}

In order to study the linear stability of DissNLS solutions of the form given in equation (6) with $\omega_{r}(t)$ and $\omega_{i}(t)$ given in equation (9), we consider perturbed solutions of the form

$\psi_{p}(x, y, t)=\left(\psi_{0}+\epsilon u(x, y, t)+i \epsilon v(x, y, t)+\mathcal{O}\left(\epsilon^{2}\right)\right) \exp \left(i k x+i l y+\omega_{r}(t)+i \omega_{i}(t)+i \xi\right)$.

Note that the decay due to $\omega_{r}(t)$ has been factored out. In essence, this allows the evolution of the perturbations $u$ and $v$ to be directly compared to the constant $\psi_{0}$. If $u$ and/or $v$ grow without bound in $t$, we say that the solution is linearly unstable (even though the magnitude of the entire perturbed solution, $\left|\psi_{p}\right|$, may decay to zero). If there do not exist any $u$ or $v$ that grow without bound, then we say that the corresponding solution is linearly stable.

Substituting equation (16) into equation (2), linearizing, and separating into real and imaginary parts leads to a pair of coupled PDEs that have constant coefficients in $x$ and $y$. Without loss of generality, assume

$$
\begin{aligned}
& u(x, y, t)=U(t) \mathrm{e}^{i p x+i q y}+c . c ., \\
& v(x, y, t)=V(t) \mathrm{e}^{i p x+i q y}+c . c .,
\end{aligned}
$$

where $U(t)$ and $V(t)$ are complex-valued functions and $p$ and $q$ are real constants. After substituting equation (17) into the PDEs, the system simplifies to

$$
\left(\begin{array}{l}
U \\
V
\end{array}\right)^{\prime}=\mathbf{A}\left(\begin{array}{l}
U \\
V
\end{array}\right)+\mathbf{B}(t)\left(\begin{array}{l}
U \\
V
\end{array}\right),
$$

where the constant matrix $\mathbf{A}$ and $\mathbf{B}(t)$ are defined by

$$
\begin{gathered}
\mathbf{A}=\left(\begin{array}{cc}
-a p^{2}-b q^{2}-2 i \alpha k p-2 i \beta l q & \alpha p^{2}+\beta q^{2} \\
-\alpha p^{2}-\beta q^{2} & -a p^{2}-b q^{2}-2 i \alpha k p-2 i \beta l q
\end{array}\right) \\
\mathbf{B}(t)=\frac{2 \psi_{0}^{2}}{1+2 c \psi_{0}^{2} t}\left(\begin{array}{rr}
-c & 0 \\
\gamma & 0
\end{array}\right) .
\end{gathered}
$$

If $\alpha p^{2}+\beta q^{2}=0$, then the solution to the system in equation (18) is

$$
\begin{gathered}
U(t)=\frac{c_{1}}{1+2 c \psi_{0}^{2} t} \exp \left(\frac{\left(-a p^{2}-b q^{2}-2 i \alpha k p-2 i \beta l q\right)\left(1+2 c \psi_{0}^{2} t\right)}{2 c \psi_{0}^{2}}\right), \\
V(t)=-\frac{\gamma}{c} U(t)+c_{2} \mathrm{e}^{-t\left(a p^{2}+b q^{2}+2 i \alpha k p+2 i \beta l q\right)},
\end{gathered}
$$

where $c_{1}$ and $c_{2}$ are complex constants. Since $c>0$ in this case, neither the magnitude of $U(t)$ nor the magnitude of $V(t)$ increases. 
If $\alpha p^{2}+\beta q^{2} \neq 0$, then the system given in equation (18) has the following solution

$$
\begin{gathered}
U(t)=\exp \left(\frac{\left(-a p^{2}-b q^{2}-i\left(\alpha p^{2}+2 \alpha k p+\beta q^{2}+2 \beta l q\right)\right)\left(1+2 c \psi_{0}^{2} t\right)}{2 c \psi_{0}^{2}}\right)\left(1+2 c \psi_{0}^{2} t\right) * \\
\left(c_{1} \mathcal{U}\left(\frac{3 c-i \gamma}{2 c}, 3, z\right)+c_{2} \mathcal{L}_{\frac{-3 c+i \gamma}{2 c}}^{2}(z)\right), \\
(21 \mathrm{a}) \\
V(t)=\frac{i\left(1+2 c \psi_{0}^{2} t\right)}{\alpha p^{2}+\beta q^{2}} \exp \left(\frac{\left(-a p^{2}-b q^{2}-i\left(\alpha p^{2}+2 \alpha k p+\beta q^{2}+2 \beta l q\right)\right)\left(1+2 c \psi_{0}^{2} t\right)}{2 c \psi_{0}^{2}}\right) * \\
\left(\frac{c_{1}\left(\alpha p^{2}+\beta q^{2}\right)(i \gamma-3 c)}{c} \mathcal{U}\left(\frac{5 c-i \gamma}{2 c}, 4, z\right)-c_{1}\left(\alpha p^{2}+\beta q^{2}+\frac{4 i c \psi_{0}^{2}}{1+2 c \psi_{0}^{2} t}\right) \mathcal{U}\left(\frac{3 c-i \gamma}{2 c}, 3, z\right)\right. \\
\left.-2 c_{2}\left(\alpha p^{2}+\beta q^{2}\right) \mathcal{L}_{\frac{-5 c+i \gamma}{2 c}}^{3}(z)+c_{2}\left(\alpha p^{2}+\beta q^{2}+\frac{4 i c \psi_{0}^{2}}{1+2 c \psi_{0}^{2} t}\right) \mathcal{L}_{\frac{-3 c+i \gamma}{2 c}}^{2}(z)\right)
\end{gathered}
$$

where $z=\left(\alpha p^{2}+\beta q^{2}\right)\left(1+2 c \psi_{0}^{2} t\right) /\left(c \psi_{0}^{2}\right), \mathcal{U}$ is the confluent hypergeometric function of the second kind and $\mathcal{L}$ is the associated Laguerre polynomial [1]. The magnitudes of $U(t)$ and $V(t)$ decay to zero (while oscillating) because the arguments of $\mathcal{U}$ and $\mathcal{L}$ are purely imaginary. Therefore, since there do not exist any $u$ or $v$ that grow without bound, all plane-wave solutions defined by equation (9) are linearly stable.

\subsection{Cases 3 and 4: $c \geq 0$ and $a k^{2}+b l^{2}+d>0$}

In this section, we consider the stability of the case 3 and case 4 solutions simultaneously. In order to study the stability of these solutions, we consider perturbed solutions of the form given in equation (16) with $\omega_{r}$ and $\omega_{i}$ defined in equation (10) or (11). Substituting the perturbed solution into the DissNLS equation and assuming that $u$ and $v$ are defined in equation (17) leads to

$$
\left(\begin{array}{l}
U \\
V
\end{array}\right)^{\prime}=\mathbf{A}\left(\begin{array}{l}
U \\
V
\end{array}\right)+\mathbf{B}(t)\left(\begin{array}{l}
U \\
V
\end{array}\right),
$$

where the constant matrix $\mathbf{A}$ and $\mathbf{B}(t)$ are given by

$$
\begin{gathered}
\mathbf{A}=\left(\begin{array}{cc}
-a p^{2}-b q^{2}-2 i \alpha k p-2 i \beta l q & \alpha p^{2}+\beta q^{2}-2 i a k p-2 i b k p \\
\left.-\alpha p^{2}-\beta q^{2}+2 i a k p+2 i b k p-a p^{2}-b q^{2}-2 i \alpha k p-2 i \beta l q\right)
\end{array}\right) \\
\mathbf{B}(t)=2 \psi_{0}^{2} \mathrm{e}^{-2 t\left(a k^{2}+b l^{2}+d\right)}\left(\begin{array}{cc}
-c & 0 \\
\gamma & 0
\end{array}\right) .
\end{gathered}
$$


To our knowledge, an analytic solution of the system given in equation (22) is not known. However, the large- $t$ solution of this system behaves as the $\mathbf{B}(t)=$ $\mathbf{0}$ case because $\mathbf{B}(t)$ decays exponentially and is integrable on $t \in[0, \infty)[8,9]$. Therefore, thee stability of the case 3 and case 4 solutions is determined by the eigenvalues of $\mathbf{A}$.

The eigenvalues of $\mathbf{A}$ are

$$
\begin{gathered}
\lambda_{1}=-a p(2 k+p)-b q(2 l+q)-i(\alpha p(2 k+p)+\beta q(2 l+q)), \\
\lambda_{2}=a p(2 k-p)+b q(2 l-q)-i(\alpha p(2 k-p)+\beta q(2 l-q)) .
\end{gathered}
$$

If either $\lambda_{1}$ or $\lambda_{2}$ has positive real part, then the magnitudes of $U$ and $V$ will grow exponentially in $t$ (after a transient period during which $\mathbf{B}(t)$ may effect $U$ and $V$ ) and the underlying DissNLS solution is said to be linearly unstable. If both $\lambda_{1}$ and $\lambda_{2}$ have nonpositive real parts, then the perturbations do not grow in $t$ (after the transient period) and then underlying DissNLS solution is said to be linearly stable.

If $a=b=0$ (forcing $d>0$ to remain in case 3 or 4 ), then both $\lambda_{1}$ and $\lambda_{2}$ have zero real part. Therefore, all plane-wave solutions of the DissNLS equation (regardless of their spatial dependence) are linearly stable if $a=b=0$ and $d>0$. This result is consistent with the results in [22].

If the underlying DissNLS solution is spatially independent $(k=l=0)$, then both $\lambda_{1}$ and $\lambda_{2}$ have nonpositive real parts. This establishes that all such solutions are linearly stable.

Any choice of $p$ and $q$ for which

$$
a p^{2}+b q^{2}<-2(a k p+b l q) \text { or } a p^{2}+b q^{2}<2(a k p+b l q),
$$

leads to an eigenvalue with positive real part. This establishes that all spatiallydependent solutions in this case are linearly unstable if $a$ and $b$ are positive. The maximum growth rate is given by

$$
\max _{p, q} \Re\left(\lambda_{1}, \lambda_{2}\right)=a k^{2}+b l^{2}
$$

and is achieved if $p=-k$ and $q=-l$. This establishes that the most unstable mode is the $(p, q)=(-k,-l)$-mode. Distributing the exponential term in equation (16) demonstrates that the most unstable mode corresponds to the $(0,0)$-mode (the mode with the overall slowest decay rate). Simply put, the linear theory picks out the mode with the overall slowest rate of decay.

\subsection{Summary}

- If $c$ and/or $d$ is positive, then regardless of the signs of $\alpha, \beta$, and $\gamma$ and values 
of $a, b \geq 0$, all spatially-independent plane-wave solutions of the DissNLS equation are linearly stable.

- If $a=b=0$ and if $c$ and/or $d$ is positive, then all (both spatially-independent and spatially-dependent) plane-wave solutions of the DissNLS equation are linearly stable.

- If $a>0$, then regardless of the signs of $\alpha, \beta$, and $\gamma$ and values of $b, c, d \geq 0$, all plane-wave solutions of the DissNLS equation with $x$-spatial dependence are linearly unstable.

- If $b>0$, then regardless of the signs of $\alpha, \beta$, and $\gamma$ and values of $a, c, d \geq 0$, all plane-wave solutions of the DissNLS equation with $y$-spatial dependence are linearly unstable.

- If $a k^{2}+b l^{2}>0$, then the perturbation corresponding to any choice of $(p, q)$ satisfying $a p^{2}+b q^{2}<-2(a k p+b l q)$ or $a p^{2}+b q^{2}<2(a k p+b l q)$ will grow exponentially relative to $\psi_{0}$ after a transient period.

- If instability exists, then the most unstable mode is the $(p, q)=(-k,-l)$ mode and the maximum growth rate is $a k^{2}+b l^{2}$.

- If $c>0$ and/or $d>0$, then the magnitude of all DissNLS (both perturbed and unperturbed) solutions decays to zero in $t$ because the decay rate due to $\omega_{r}(t)$ is greater than the maximum growth rate of $u(x, y, t)$ and $v(x, y, t)$.

- All of the above results and observations are valid in the one-dimensional case (i.e. $\beta=b=l=q=0$ ).

- All of the above results and observations generalize to the three-dimensional version of the DissNLS equation.

\section{$3 \quad$ Numerical simulations}

In order to corroborate the theoretical results in the previous section and to examine the effect of the transient period that arises in case 3 and 4 , we numerically solved the systems given in equations (2), (18), and (22) for a wide range of parameter values. We solved the DissNLS equation numerically using a split-step pseudospectral routine $[25,12,7]$. Although all simulations produced results consistent with the theory, we only present results from a few simulations here.

\subsection{Case \#2 simulations}

We considered the DissNLS equation with $\alpha=\beta=\gamma=1, a=b=d=0$, $c=1$ and initial condition

$$
\psi(x, y, 0)=\left(1+0.1 \mathrm{e}^{-2 i x+5 i y}\right) \mathrm{e}^{2 i x-5 i y}=\mathrm{e}^{2 i x-5 i y}+0.1
$$

For this initial condition, $k=2, l=-5, p=-2, q=5$, and $\psi_{0}=1$. 
(a)

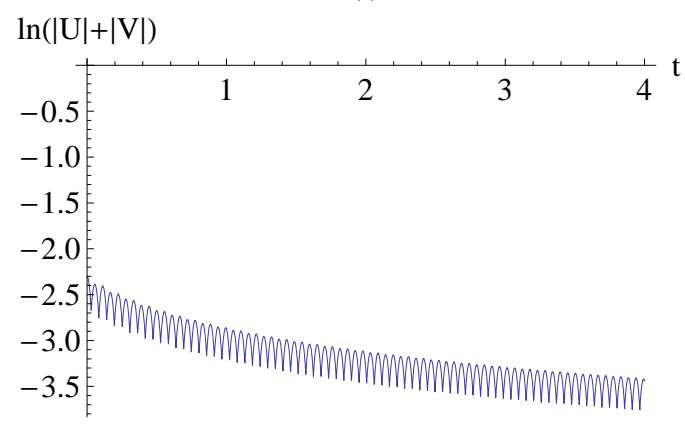

(b)

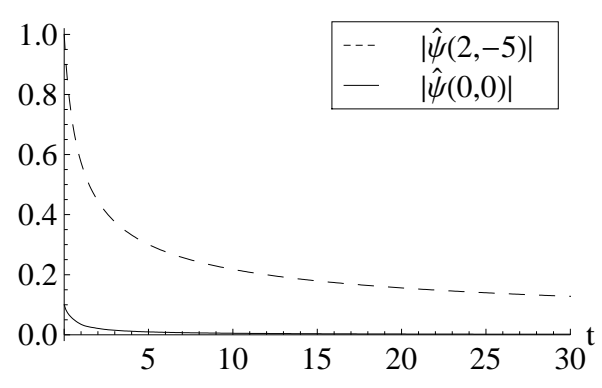

Fig. 1. Results from numerical simulations of (a) the system given in equation (18) and (b) equation (2). For both plots, $\alpha=\beta=\gamma=1, a=b=d=0, c=1$ and the initial condition given in equation (27).

The plot in Figure 1(a) was obtained by numerically solving the system given in equation (18). This plot demonstrates that the magnitudes of the perturbations $U(t)$ and $V(t)$ decrease (while oscillating) in $t$. Figure 1(b) was obtained by numerically solving the DissNLS equation. It contains plots of the magnitudes of the $(2,-5)$ - and $(0,0)$-modes versus $t$. The magnitude of both modes decay to zero. The $(2,-5)$-mode remains dominant for all $t \geq 0$. These simulations establish that this plane-wave solution is stable with respect to this perturbation just as predicted in Section 2.2.

\subsection{Case \#4 simulations}

For this case, we considered $\alpha=\beta=\gamma=1, a=b=c=d=0.1$ and the initial condition given in equation (27). The perturbation in this case is the most unstable perturbation. Figure 2(a) was obtained by solving the system in equation (22). The plot demonstrates that $\ln (|U|+|V|)$ increases with a rate of 0.289 , nearly the rate of 0.29 predicted in equation (26). Figure 2(b) was obtained by numerically solving the DissNLS equation. It demonstrates that the magnitudes of both modes decay to zero, but the magnitude of the $(2,-5)$ mode decays with a much faster rate. At approximately $t=8$, the $(0,0)$-mode becomes the dominant mode. Note that the transient period does not play a significant role in either simulation. Among other things, these plots corroborate the prediction that spatially-dependent solutions are unstable with respect to perturbations that satisfy equation (25).

Next we considered the following initial condition in the DissNLS equation with the same equation parameters

$$
\psi(x, y, 0)=\left(1+0.1 \mathrm{e}^{3 i x+2 i y}\right) \mathrm{e}^{2 i x-5 i y}=\mathrm{e}^{2 i x-5 i y}+0.1 \mathrm{e}^{5 i x-3 i y} .
$$

For this initial condition, $k=2, l=-5, p=3, q=2$, and $\psi_{0}=1$. The 
(a)

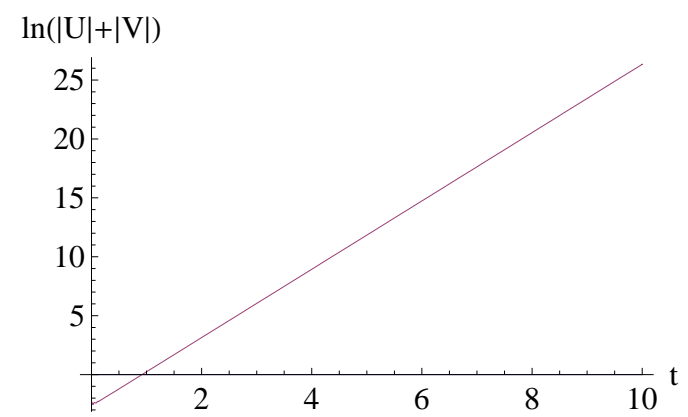

(b)

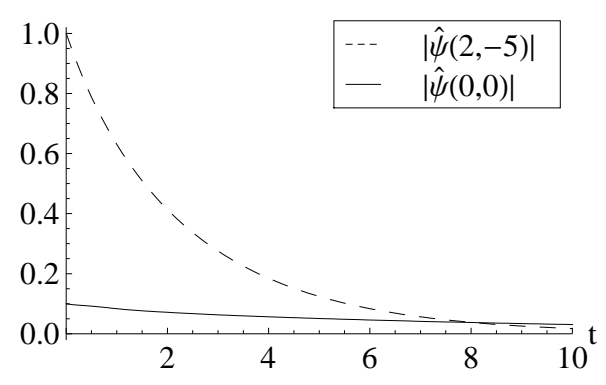

Fig. 2. Results from numerical simulations of (a) the system given in equation (22) and (b) equation (2). For both plots, $\alpha=\beta=\gamma=1, a=b=c=d=0.01$ and the initial condition is given in equation (28).

(a)

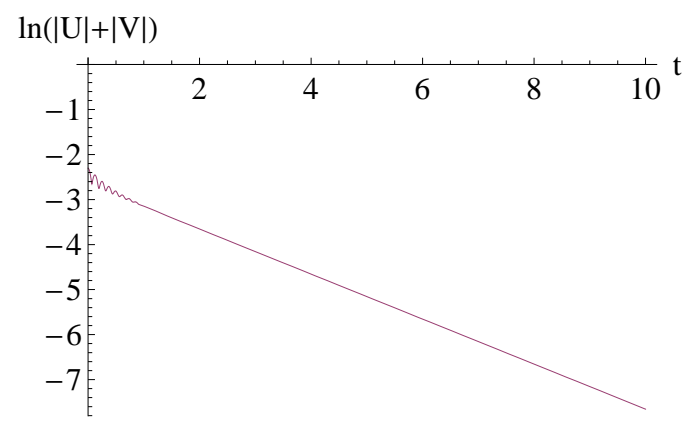

(b)

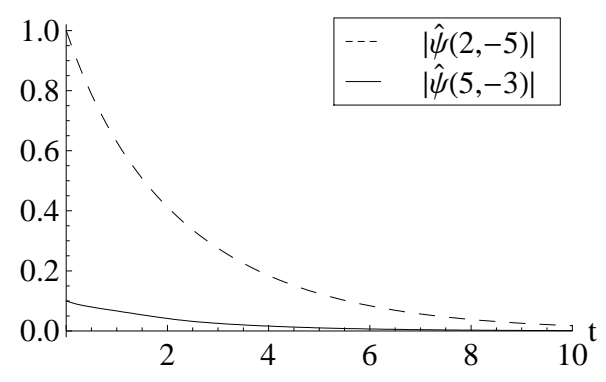

Fig. 3. Results from numerical simulations of (a) the system given in equation (18) and (b) equation (2). For both plots, $\alpha=\beta=\gamma=1, a=b=c=d=0.01, c=1$ and the initial condition is given in equation (28).

perturbation in this case is predicted to decay to zero.

Figure 3(a) was obtained by numerically solving the system given in equation (22). In this case, $\ln (|U|+|V|)$ decays. Notice that the behavior of the solution during the transient period is qualitatively similar to the large- $t$ solution behavior. Further, note that the magnitudes of the perturbations decrease exponentially. Figure 3(b) was obtained by numerically solving the DissNLS equation. In this case, the magnitudes of both modes decay to zero, but the magnitude of the $(2,-5)$-mode decays with a slower rate and remains the dominant mode for all $t \geq 0$. This corroborates the prediction that spatiallydependent solutions are stable with respect to perturbations that do not satisfy equation (25). Note that the $(0,0)$-mode (the mode with the slowest overall rate of decay) does not play a role in this simulation because it was not seeded as part of the initial condition and its magnitude remains negligible.

\section{Numerical Observations:}


- If $d>0$ or if $c>0$, then the magnitude of all solutions decays to zero.

- If an initial condition of the form given in equation (27) is used, then the $(0,0)$-mode eventually becomes dominant because the spatially-dependent initial condition is unstable.

- If an initial condition of the form given in equation (28) is used, then the mode corresponding to the unperturbed solution remains dominant because the perturbations do not satisfy equation (25).

This material is based upon work supported by the National Science Foundation under grant DMS-0139771. We acknowledge helpful discussions with Frederic Dias, Harvey Segur, Diane Henderson, and Thomas Bridges.

\section{References}

[1] M. Abramowitz and I.A. Stegun. Handbook of Mathematical Functions. Dover, 1972.

[2] I.S. Aranson and L. Kramer. The world of the complex Ginzburg-Landau equation. Reviews of Modern Physics, 74:99-143, 2002.

[3] D.J. Benney and G.J. Roskes. Wave instabilities. Studies in Applied Mathematics, 48(377), 1969.

[4] P.J. Blennerhassett. On the generation of waves by wind. Philosophical Transactions of the Royal Society of London. Series A, Mathematical and Physical Sciences, 298(1441):451-494, 1980.

[5] T.J. Bridges and F. Dias. Enhancement of the Benjamin-Feir instability with dissipation. Physics of Fluids, 19:104104, 2007.

[6] R. Brown, A.L. Fabrikant, and M.I. Rabinovich. Evolution of patterns in the anisotropic complex Ginzburg-Landau equation: Modulational instability. Physical Review E, 47(6):4141-4150, 1993.

[7] J.D. Carter. Stability and existence of traveling wave solutions of the twodimensional nonlinear Schrödinger equation and its higher-order generalizations. PhD thesis, University of Colorado at Boulder, 2001.

[8] E.A. Coddinton and N. Levinson. Theory of Ordinary Differential Equations. McGraw-Hill, New York, 1955.

[9] W.A. Coppel. Stability and Asymptotic Behavior of Differential Equations. Heath, Boston, 1965.

[10] A. Davey. The propagation of a weak nonlinear wave. Journal of Fluid Mechanics, 53:769-781, 1972.

[11] A. Davey and K. Stewartson. On three-dimensional packets of surface waves. Proceedings of the Royal Society Series A, 338:101-110, 1974.

[12] B. Fornberg. A Practical Guide to Pseudospectral Methods. Cambridge University Press, 1996.

[13] V.L. Ginzburg and L.D. Landau. On the theory of superconductivity. Zhurnal Eksperimental'noi i Teoreticheskoi Fiziki, 20:1064, 1950. 
[14] E.P. Gross. Hydrodynamics of a superfluid condensate. Journal of Mathematical Physics, 4(2), February 1963.

[15] B.M. Lake, H.C. Yuen, H. Rungaldier, and W.E. Ferguson. Nonlinear deep-water waves: theory and experiment. Part 2. Evolution of a continuous wave train. Journal of Fluid Mechanics, 83:49-74, 1977.

[16] S.V. Manakov. On the theory of two-dimensional stationary self-focusing of electromagnetic waves. Soviet Physics JETP, 38, 1974.

[17] J.W. Miles. Surface-wave damping in closed basins. Proceedings of the Royal Society London A, 297:459-475, 1967.

[18] A.C. Newell and J.A. Whitehead. Finite bandwidth, finite amplitude convection. Journal of Fluid Mechanics, 38(279-303), 1969.

[19] H.L. Pecseli. Solitons and weakly nonlinear waves in plasmas. IEEE Transactions on Plasma Science, 13(2):53-86, April 1985.

[20] L.P. Pitaevskii. Vortex lines in an imperfect Bose gas. Soviet Physics JETP, 13(2), August 1961.

[21] Z. Rapti, P.G. Kevrekidis, D.J. Frantzeskakis, and B.A. Malomed. On the modulational instability of the nonlinear Schrödinger equation with dissipation. Physica Scripta, T113:74-77, 2004.

[22] H. Segur, D. Henderson, J.D. Carter, J. Hammack, C. Li, D. Pheiff, and K. Socha. Stabilizing the Benjamin-Feir instability. Journal of Fluid Mechanics, 539:229-271, 2005.

[23] J.T. Stuart and R.C. DiPrima. Eckhaus and Benjamin-Feir resonance mechanisms. Proceedings of the Royal Society Series A, 362:27-41, 1978.

[24] G. Wu, Y. Lui, and D.P. Yue. A note on stabilizing the Benjamin-Feir instability. Journal of Fluid Mechanics, 556:45-54, 2006.

[25] H. Yoshida. Construction of higher order symplectic integrators. Physics Letters A, 150:262-268, 1990.

[26] V.E. Zakharov. Stability of periodic waves of finite amplitude on the surface of a deep fluid. Soviet Physics Journal of Applied Mechanics and Technical Physics, 4:86-94, 1968. 